\title{
РЕЗОНАНСНОЕ КОМБИНАЦИОННОЕ РАССЕЯНИЕ С УЧЕТОМ ЭФФЕКТА ЯНА-ТЕЛЛЕРА; СВЯЗЬ С ПОГЛОЩЕНИЕМ
}

\section{1. Введение}

Спектральные и поляризационные характеристики резонансного комбинационного рассеяния (РКР) примесных центров кристаллов и молекул в растворах зависят от вибронного взаимодействия, вид которого определяется симметрией резонансных электронных состояний. В центрах (молекулах) низкой симметрии электронные состояния не вырождены и в линейном приближении отличный от нуля вклад в вибронное взаимодействие дают только полносимметричные колебания. В центрах высокой симметрии часть электронных состояний вырождена. В этих состояниях согласно теореме Яна-Теллера [ $\left.{ }^{1}\right]$ в линейном приближении в вибронное взаимодействие дают вклад и неполносимметричные колебания.

В вырожденных состояниях многомодовых систем аналитическое рассмотрение PKР с учетом эффекта Яна-Теллера в общем случае оказывается невозможным. Приближенное решение задачи может быть найдено, если вибронное взаимодействие по неполносимметричным недиагональным * колебаниям либо сильное, либо слабое. В первом случае выполняется условие $\sigma_{n d} \gg \bar{\omega}\left(\sigma_{n d}-\right.$ вклад отмеченных колебаний в квадратичную дисперсию полөсы поглощения, $\bar{\omega}-$ средняя частота колебаний). Наличие малого параметра $\bar{\omega} / \sigma_{n d}$ позволяет использовать полуклассическое приближение, хорошо известное в теории спектров поглощения и люминесценции. Теория РКР в этом приближении развита в работах $[2,3]$. Здесь рассматривается случай слабого взаимодействия оптических электронов с неполносимметричными недиагонаяьными (трансверсальными) колебаниями, но вклад остальных - как полносимметричных, так и неполносимметричных диагональных (радиальных) колебаний учитываетея точно.

Конкретно будут рассчитаны тензоры (амплитуды) рассеяния. Квадрат их дает возбуждающий профиль РКР на данной моде - зависимость сечения РКР от частоты возбуждения $\omega_{0}$ в области полосы поглощения при сохранении разности $\omega_{1 j}=\omega_{0}-\Omega$ возбуждающей $\omega_{0}$ и рассеянной $\Omega$ частот $\left(\omega_{1 j}\right.$ - частота исследуемой моды в основном электронном состоянии). Ввиду того, что разные колебания, участвующие в процессе рассеяния, имеют разные, характерные для данной моды возбуждающие профили, РКР оказывается гораздо более информативным, чем поглощение.

Для моделирования наблюдаемых возбуждающих профилей РКР ниже предлагается метод, сводящий их вычисление к преобразованию спектра поглощения центра, возмущенного внешним полем. Этот метод

* Эти колебания дают недиагональный вклад в матрищу вибронного взаимодействия, 
является обобщением метода преобразөвания [4-6] на случай вырожденных возбужденных электронных состояний. Предлагаемый метод применим не только в случае вырожденных, но и в случае близких электронных уровней.

\section{2. Расчет оператора поляризуемости} $\left.\left[{ }^{7}\right]\right)$

Исходим из следующей формулы для сечения рассеяния (см. напр.,

$$
I\left(\omega_{0}, \Omega\right)=\sum_{\substack{\alpha \beta \\ \alpha^{\prime} \beta^{\prime}}} i_{\alpha^{\prime}} i_{\alpha^{\prime}} j_{\beta} j_{\beta}{ }^{\prime} W_{\alpha \beta \beta^{\prime} \alpha^{\prime}}\left(\omega_{0}, \Omega\right),
$$

где $i_{\alpha}, j_{\beta}-$ декартовы компоненты единичных векторов поляризации возбуждающего и рассеянного света, а тензор вторичного свечения $W_{\alpha \beta \beta^{\prime} \alpha^{\prime}}\left(\omega_{0}, \Omega\right)$ представлен в ковариантном виде

$$
W_{\alpha \beta \beta^{\prime} \alpha^{\prime}}\left(\omega_{0}, \Omega\right)=\frac{1}{2 \pi} \int_{-\infty}^{\infty} d t e^{i\left(\omega_{0}-\Omega\right) t}\left\langle P_{\alpha \beta}(t) P_{\beta^{\prime} \alpha^{\prime}}^{+}\right\rangle,
$$

удобном для применения корреляционной техники. Здесь

$$
P_{\alpha \beta}=i \int_{0}^{\infty} d \tau e^{-i \tau \omega_{0}-i \tau H} d_{\alpha}^{+} e^{i \tau \hat{H}} d_{\beta}
$$

- оператор поляризуемости; $\hat{H}=H+i \gamma, H-$ гамильтониан вещества, $\gamma$ - коммутирующий с $H$ оператор радиационного затухания, $\vec{d}$ - оператор дипольного момента оптических электронов; $P_{\alpha \beta}(t)=\exp (i t H) P_{\alpha \beta} \exp (-i t H) ;\langle\ldots\rangle=\mathrm{Sp}\left(e^{-H / k T} \ldots\right) / \mathrm{Sp}\left(e^{-H / k T}\right)$

означают квантовомеханическое и статистическое усреднения.

Формулы (1)-(3) являются общими. Их можно существенно упростить, если воспользоваться адиабатическим приближением. Если исходное (основное) электронное состояние $|1\rangle$ достаточно удалено от возбужденных (по сравнению с $k T$ ), то в (2) усреднение можно проводить лишь по колебательным состояниям электронного состояния |1). Если, кроме того, конечное электронное состояние совпадает с начальным, то оператор поляризуемости принимает вид

$$
P_{\alpha \beta}=\sum_{e} P_{\alpha \beta}^{(e)}
$$

где

$$
P_{\alpha \beta}^{(e)}=i \int_{0}^{\infty} d \tau e^{-i \tau \omega_{0}-i \tau H_{1}} M_{\alpha, e}^{+} e^{i \tau H_{e}-\nu_{e} \tau} M_{\beta, e}
$$

- вклад возбужденного электронного уровня $|e\rangle$ в оператор поляризуемости исходного электронного состояния $|1\rangle . H_{1}\left(H_{e}\right)$ - колебательный гамильтониан основного (возбужденного) электронного состояния; в предположении $n$-кратного вырождения электронного состояния $|e\rangle$ $H_{e}=H_{1}+V$ - матрица порядка $n \times n, \gamma_{e}$ - оператор радиационного затухания $|e\rangle, M_{\alpha, e}=\left\langle e\left|d_{\alpha}\right| 1\right\rangle$ - электронный матричный элемент дипольного перехода $|1\rangle \rightarrow|e\rangle$.

В дальнейшем считается, что электронная часть гамильтониана $H_{e}$ диагонализована в базисе состояний $|\alpha\rangle$. Тогда в приближении Кондона оператор $P_{\alpha \beta}$ выражается через линейную комбинацию операторов

$$
P_{\alpha \beta}=i \int_{0}^{\infty} d \tau e^{-i \tau \omega_{0}-i \tau H_{1}} e^{-\gamma_{\beta} \tau}\left(e^{i \tau H_{e}}\right)_{\alpha \beta}
$$


Следует отметить, что переменная интегрирования $\tau$ в формулах (4), (5) может быть интерпретирована как время пребывания амплитуды состояния в возбужденном электронном состоянии.

В случае вырожденных электронных состояний основная трудность при вычислении $P_{\alpha \beta}$ связана с некоммутируемостью матриц $V$ и $\left[H_{1}, V\right]$ в матричном операторе $H_{e}=H_{1}+V$, связанной с недиагональностью матрицы $V$. Мы предположим, что в вибронном базисе недиагональная часть $V$ мала: $V_{\alpha \beta} \ll V_{\alpha \alpha}$ (взаимодействие с неполносимметричричными колебаниями, дающими недиагональный вклад в $V$, мало́). Основой такого предположения являются классические представления, по которым вибронное взаимодействие с радиальными колебаниями сильнее, чем с трансверсальными. В этом случае матрицу $\exp \left(i \tau H_{e}\right)$ можно разложить в ряд, используя теорему $\left[{ }^{8}\right]$

$$
\begin{gathered}
\exp (a+b)=(\exp a) T \exp \left\{i \int_{0}^{1} d s b(-s)\right\}, \\
b(-s)=\exp (-a s) b \exp (a s) .
\end{gathered}
$$

Знак $T$ при экспоненте означает хронологическое упорядочение. Основываясь на $(6)$, представим $\exp \left(i \tau H_{e}\right)$ в виде произведения диагональной $\left(\exp \left(i \tau H_{\alpha \alpha}\right)\right)$ и недиагональной $\left(S_{\alpha \beta}\right)$ частей

$$
\left(\exp \left(i \tau H_{e}\right)\right)_{\alpha \beta}=\exp \left(i \tau H_{\alpha \alpha}\right) \bar{S}_{\alpha \beta}(\tau),
$$

где недиагональная часть определена упорядоченной экспонентой

$$
\begin{gathered}
\bar{S}_{\alpha \beta}(\tau)=T \exp \left\{i \int_{0}^{\tau} d s \bar{V}_{\alpha \beta}(-s)\right\}, \\
\bar{V}_{\alpha \beta}(-s)=\exp \left(-i s H_{\alpha \alpha}\right) V_{\alpha \beta} \exp \left(i s H_{\beta \beta}\right) .
\end{gathered}
$$

Диагональная часть гамильтониана возбужденного электронного состояния $H_{\alpha \alpha}$ связана с гамильтонианом основного электронного состояния $H_{1}$ аналогично $(6),(7)$ :

$$
\begin{array}{r}
\exp \left(i \tau H_{\alpha \alpha}\right)=\exp \left(i \tau H_{1}\right) S_{\alpha \alpha}(0, \tau), \\
S_{\alpha \alpha}\left(\tau, \tau^{\prime}\right)=T \exp \left\{i \int_{\tau}^{\tau^{\prime}} d s V_{\alpha \alpha}(-s)\right\},
\end{array}
$$

где $V_{\alpha \alpha}(-s)$ - диагональные элементы матрицы вибронного взаимодействия в представлении Гейзенберга по гамильтониану осыовного состояния

$$
V_{\alpha \alpha}(-s)=\exp \left(-i s H_{1}\right) V_{\alpha \alpha} \exp \left(i s H_{1}\right)
$$

Рассматривая недиагональные элемекты $V_{\alpha \beta}$ матрицы вибронного взаимодействия как малые, получим следующие приближенные формулы для диагональных и недиагональных компонент оператора поляризуемости

$$
\begin{aligned}
& P_{\alpha \alpha}^{(0)} \simeq i \int_{0}^{\infty} d \tau \mathrm{e}^{-i \tau \omega_{0}-\gamma_{\alpha} \tau} S_{\alpha \alpha}(0, \tau) \\
& P_{\alpha \beta}^{(1)} \simeq \int_{0}^{\infty} d \tau e^{-i \tau \omega_{0}-\gamma_{\beta} \tau} \int_{0}^{\tau} d s S_{\alpha \alpha}(s, \tau) V_{\alpha \beta}(-s) S_{\beta \beta}(0, s),
\end{aligned}
$$

причем переменная интегрирования $s$ имеет смысл времени перехода амплитуды из состояния исходной поляризации $\alpha$ в конечную $\beta$. 


\section{3. Связь амплитуды рассеяния со спектром поглощения}

Спектр поглощения в базисе состояний $|\alpha\rangle$, диагонализующем электронную энергию, определяется формулой

$$
\varkappa_{\alpha \beta}\left(\omega_{0}\right)=\frac{1}{2 \pi} \int_{-\infty}^{\infty} d \tau e^{-i \tau \omega_{0}-\gamma_{\beta}|\tau|}\left\langle e^{-i \tau H_{1}}\left(e^{i \tau H_{e}}\right)_{\alpha \beta}\right\rangle .
$$

Используя при расчете коррелятора разложение (8) с предположением $V_{\alpha \beta} \ll V_{\alpha \alpha}$, получим в нулевом приближении

$$
\begin{aligned}
x_{\alpha \alpha}^{(0)}\left(\omega_{0}\right) & =\frac{1}{2 \pi} \int_{-\infty}^{\infty} d \tau e^{-i \tau \omega_{0}-v_{\alpha}|\tau|}\left\langle e^{-i \tau H_{1}} e^{i \tau H_{\alpha \alpha}}\right\rangle= \\
& =\frac{1}{2 \pi} \int_{-\infty}^{\infty} d \tau e^{-i \tau \omega_{0}-v_{\alpha}|\tau|}\left\langle S_{\alpha \alpha}(0, \tau)\right\rangle,
\end{aligned}
$$

а в первом приближении

$$
\begin{gathered}
x_{\alpha \beta}^{(1)}\left(\omega_{0}\right)=\frac{i}{2 \pi} \int_{-\infty}^{\infty} d \tau e^{-i \tau \omega_{0}-\gamma_{\beta}|\tau|} \int_{0}^{\tau} d s\left\langle V_{\alpha \beta}(-s)\right\rangle \times \\
\quad \times\left\langle S_{\alpha \alpha}(s, \tau) S_{\beta \beta}(0, s)\right\rangle .
\end{gathered}
$$

Поправка $x_{\alpha \beta}^{(1)}$ равна нулю, если отсутствуют внешние поля и вибронное взаимодействие учтено в линейном приближении (в этом случае $\left.\left\langle V_{\alpha \beta}\right\rangle=0\right)$. Однако, если имеется низкосимметричное внешнее поле, то $\left\langle V_{\alpha \beta}\right\rangle \neq 0$ и $x_{\alpha \beta}^{(1)} \sim\left\langle V_{\alpha \beta}\right\rangle \neq 0$.

Если ограничиться низкими температурами, то можно пренебречь вкладом виртуальных фононов в процессе рассеяняя [9 $]$. Тогда температурное усреднение в тензоре вторичного свечения (2) сводится к температурному усреднению амплитуд *, причем РКР первого порядка на моде $j$ определяется амплитудой $\left[{ }^{10,11}\right]$

$$
A_{\alpha \beta, j}\left(\omega_{0}\right)=\left\langle P_{\alpha \beta} a_{1 j}^{+}\right\rangle\left(\bar{n}_{j}+1\right)^{-1 / 2} .
$$

Здесь учтено, что $\left\langle n_{j}\left|P_{\alpha \beta}\right| n_{j}+1\right\rangle=\left\langle n_{j}\left|P_{\alpha \beta} a_{1 j}^{+}\right| n_{j}\right\rangle, a_{1 j}^{+}-$оператор рождения фонона в основном электронном состоянии, $n_{j}=$ $=\left[\exp \left(\omega_{1 j} / k T\right)-1\right]^{-1}, \omega_{1 j}-$ частота моды $j$ в основном электронном состоянии.

Возбуждающий профиль РКР определяется квадратом амплитуды

$$
I_{\alpha \beta \beta^{\prime} \alpha^{\prime}}\left(\omega_{0}\right)=\left|A_{\alpha \beta, j}\left(\omega_{0}\right)\right|^{2} .
$$

\section{а. РКР на диагональных колебаниях}

Рассмотрим прежде всего рассеяние параллельной поляризации (поляризации рассеянного света $\vec{j}$ и возбуждающего света $\vec{i}$ параллельны $\vec{j} \| \vec{i}) I_{\alpha \alpha \beta \beta}\left(\omega_{0}\right)$. Оно определено диагональной компонентой оператора поляризуемости $P_{\infty x}^{(0)}(13)$

$$
\left\langle P_{\alpha \alpha}^{(0)} a_{1 j}^{+}\right\rangle=i \int_{0}^{\infty} d \tau e^{-i \tau \omega_{0}-v_{\alpha} \tau}\left\langle S_{\alpha \alpha}(0, \tau) a_{1 j}^{+}\right\rangle .
$$

" Законность такой процедуры обсуждаетстя подробно в [1'], 
Чัтобы получить соотношение амплитуды (20) со спектром поглощения (16), требуется дальнейшее уточнение модели. Задача решена для линейного [ $\left.{ }^{4}\right]$ и квадратичного вибронного взаимодействия $\left[{ }^{11-13}\right]$ с учетом также Герцберг-Теллеровского взаимодействия $\left[{ }^{11,14-16}\right]$. С учетом только линейного взаимодействия операторы рождения фонона в основном и возбужденном электронных состояниях $a_{1 j}^{+}$и $a_{2 j}^{+}$отличаются только константой: $a_{1 j}^{+}=a_{2 j}^{+}-\xi_{j}-$ безмерный параметр вибронного взаимодействия $\xi_{j} ; \omega_{1 j}=\omega_{2 j}=\omega_{j}$. Следовательно,

$$
\begin{aligned}
& \left\langle S_{\alpha \alpha}(0, \tau) a_{1 j}^{+}\right\rangle=\left\langle e^{-i \tau H_{1}} e^{i \tau H_{\alpha \alpha}} a_{1 j}^{+}\right\rangle= \\
& =\left\langle e^{-i \tau H_{1}} e^{i \tau H_{\alpha \alpha}}\left(a_{2 j}^{+}+\xi_{j}\right)\right\rangle= \\
& =e^{-\omega_{j} / k T}\left\langle S_{\alpha \alpha}(0, \tau) a_{1 j}^{+}\right\rangle+\xi_{j}\left(e^{i \tau \omega_{j}}-1\right)\left\langle S_{\alpha \alpha}(0, \tau)\right\rangle .
\end{aligned}
$$

Решая это простое уравнение для $\left\langle S_{\alpha \alpha}(0, \tau) a_{1 j}^{+}\right\rangle$, получим следующее соотношение между фурье-амплитудой РКР и фурье-преобразованием $F^{(0)}(\tau)=\left\langle S_{\alpha \alpha}(0, \tau)\right\rangle$ спектра поглощения

$$
\begin{gathered}
A_{\alpha \beta}(\tau)=\left(\bar{n}_{j}+1\right)^{-1 / 2}\left\langle S_{\alpha \alpha}(0, \tau) a_{1 j}^{+}\right\rangle= \\
=\xi_{j}\left(\bar{n}_{j}+1\right)^{1 / 2}\left(e^{i \tau \omega_{j}}-1\right) P^{(0)}(\tau),
\end{gathered}
$$

что дает после фурье-преобразования следующую амплитуду PKР [ $\left.{ }^{11}\right]$

$$
A_{\alpha \beta, j}\left(\omega_{0}\right)=\left(\bar{n}_{j}+1\right)^{1 / 2} \xi_{j}\left[\Phi^{(0)}\left(\omega_{0}\right)-\Phi^{(0)}\left(\omega_{0}-\omega_{j}\right)\right],
$$

где

$$
\Phi^{(0)}\left(\omega_{0}\right)=i \pi x_{\alpha \alpha}^{(0)}\left(\omega_{0}\right)+\int_{-\infty}^{\infty} d x \frac{x_{\alpha \alpha}^{(0)}(x)}{x-\omega_{0}}
$$

- комплексный показатель преломления, $\quad x_{\alpha \alpha}^{(0)}\left(\omega_{0}\right)-\quad$ спектр поглощения (16). Формула (23), полученная ранее в $\left[{ }^{4}\right]$, является главным соотношением метода преобразования $\left[{ }^{5,6}\right]$ для т. н. основной модели, не учитывающей вырождения электронных уровней и неполносимметричных колебаний. Здесь эта формула обобщена на учет вырожденных электронных уровней и вибронного взаимодействия как с полносимметричными, так и с диагональными неполносимметричными колебаниями.

Формула (22) может быть также использована для модельных расчетов возбуждающих профилей РКР: она позволяет свести указанную задачу к хорошо известной задаче расчета фурье-образа спектра поглощения. В рассматриваемой модели диагональная часть гамильтониана $H_{\alpha \alpha}$ связана с гамильтонианом основного электронного состояния $H_{1}$ оператором сдвига $\exp \nabla_{\alpha}$ :

$$
H_{\alpha \alpha}=e^{\nabla_{\alpha}} H_{1} e^{-\nabla_{\alpha}}+\varepsilon_{\alpha}
$$

$\left(\nabla_{\alpha}=\sum_{i} \xi_{0 i}^{(\alpha)} \partial / \partial \xi_{i}=\sum_{i} \xi_{0 i}^{(\alpha)}\left(a_{1 i}-a_{1 i}^{+}\right), \xi_{i}\right.$ и $\xi_{0 i}^{(\alpha)}$ - безразмерные координаты и стоксовы потери; $\varepsilon_{\alpha}$ - энергия (частота) чисто-электронного перехода). В этой модели фурье-образ спектра поглощения равен $\left[{ }^{17}\right]$

Здесь

$$
F^{(0)}(\tau)=\left\langle e^{-i \tau H_{1}} e^{\nabla_{\alpha}} e^{i \tau H_{1}} e^{-\nabla_{\alpha}}\right\rangle e^{i \tau \varepsilon_{\alpha}}=\exp \left[g_{0}(\tau)+i \tau \varepsilon_{\alpha}\right] .
$$

$$
g_{0}(\tau)=-\left\langle\nabla_{\alpha} \nabla_{\alpha}(\tau)\right\rangle+\left\langle\nabla_{\alpha}^{2}\right\rangle=\int_{-\infty}^{\infty} d \omega \xi^{2}(\omega)\left(e^{i \omega \tau}-1\right)[n(\omega)+1],
$$


где

$$
\xi^{2}(\omega)=2 \sum_{j} \xi_{0 j}^{2} \omega_{j} \delta\left(\omega^{2}-\omega_{j}^{2}\right)
$$

- спектральная функция однофононных переходов. Функция $\xi^{2}(\omega)$ может быть вычислена стандартным методом локальной динамики решетки.

\section{6. РКР на недиагональных колебаниях}

Рассеяние перпендикулярной поляризации $(\vec{j} \perp \vec{i})$ определяется компонентами тензора (2) $W_{\alpha \beta \beta \alpha}, W_{\alpha \beta \alpha \beta}$, для нахождения которых требуется вычисление коррелятора по недиагональным компонентам $P_{\alpha \beta}^{(1)}$ оператора поляризуемости (14)

$$
\left\langle P_{\alpha \beta}^{(1)} a_{1 k}^{+}\right\rangle=\int_{0}^{\infty} d \tau e^{-i \tau \omega_{0}-\gamma_{\beta} \tau} \int_{0}^{\tau} d s\left\langle S_{\alpha \alpha}(s, \tau) V_{\alpha \beta}(-s) S_{\beta \beta}(0, s) a_{1 k}^{+}\right\rangle .
$$

Повторим процедуру перестановки фононного оператора $a_{1 k}^{+}$с учетом того, что $a_{1 h}^{+}-$оператор рождения недиагонального неполносимметричного колебания, с которым линейный по таким колебаниям оператор

$$
V_{\alpha \beta}(x)=\sum_{k} c_{k}^{(\alpha \beta)}\left[a_{1 k}^{+} e^{i x \omega_{k}}+a_{1 k} e^{-i x \omega_{k}}\right]
$$

не коммутирует:

$$
\left[V_{\alpha \beta}(x), a_{1 k}^{+}\right]=c_{k}^{(\alpha \beta)} e^{-i x \omega_{k} .}
$$

Получим для рассматриваемой фурье-амплитуды РКР выражение

$$
\begin{gathered}
A_{\alpha \beta}(\tau)=\int_{0}^{\tau} d s\left\langle S_{\alpha \alpha}(s, \tau) V_{\alpha \beta}(-s) S_{\beta \beta}(0, s) a_{1 k}^{+}\right\rangle\left(\bar{n}_{k}+1\right)^{-1 / 2}= \\
=c_{k}^{(\alpha \beta)}\left(\bar{n}_{k}+1\right)^{1 / 2} \int_{0}^{\tau} d s\left\langle S_{\alpha \alpha}(s, \tau) S_{\beta \beta}(0, s)\right\rangle e^{i(\tau-s) \omega_{k} .}
\end{gathered}
$$

В рассматриваемом здесь приближении линейного вибронного взаимодействия фигурирующий в (28) и в (17) коррелятор

$$
K(s, \tau)=\left\langle S_{\alpha \alpha}(s, \tau) S_{\beta \beta}(0, s)\right\rangle
$$

равен

$$
\begin{aligned}
& K(s, \tau)=\left\langle e^{\nabla_{\alpha}(-\tau)} e^{-\nabla_{\alpha}(-s)+\nabla_{\beta}(-s)} e^{-\nabla_{\beta}}\right\rangle e^{i \tau \varepsilon_{\beta}} e^{i s\left(\varepsilon_{\alpha}-\varepsilon_{\beta}\right)}= \\
& =\exp \left\{g_{\alpha \beta}(\tau)+g_{\alpha, \alpha-\beta}(s)-g_{\alpha-\beta, \beta}(\tau-s)\right\} e^{i \tau \varepsilon_{\beta}} e^{i s\left(\varepsilon_{\alpha}-\varepsilon_{\beta}\right)},
\end{aligned}
$$

где

$$
\begin{gathered}
g_{\alpha \beta}(x)=-\left\langle\nabla_{\alpha} \nabla_{\beta}(x)\right\rangle+\left\langle\nabla_{\alpha} \nabla_{\beta}\right\rangle, \\
g_{\alpha, \alpha-\beta}(x)=-\left\langle\nabla_{\alpha}\left(\nabla_{\alpha}(x)-\nabla_{\beta}(x)\right)\right\rangle+\left\langle\nabla_{\alpha}\left(\nabla_{\alpha}-\nabla_{\beta}\right)\right\rangle .
\end{gathered}
$$

Учитывая, что

$$
g_{\alpha-\beta, \beta}=-g_{\alpha, \alpha-\beta} \equiv g_{1} \quad \text { и } \quad g_{\alpha \beta}=g_{\alpha \alpha}-g_{\alpha, \alpha-\beta} \equiv g_{0}-g_{1},
$$

формула (29) приводится к виду

$$
K(s, \tau)=\exp \left\{g_{0}(\tau)-g_{1}(\tau)+g_{1}(s)+g_{1}(\tau-s)\right\} e^{i \tau \varepsilon_{\beta}} e^{i s\left(\varepsilon_{\alpha}-\varepsilon_{\beta}\right)} .
$$


Функция $\exp \left(g_{0}(\tau)\right)$ определяет фурье-обра̋з спектра поглощения $F^{(0)}(\tau)$; она учитывает все колебания (как полно- так и неполносимметричные), дающие вклад в диагональные члены $V_{\alpha \alpha}$ вибронной матрицы $V$. Дополнительные множители $\exp \left(g_{1}(x)\right)$ учитывают только неполносимметричные диагональные колебания.

Подставляя (31) в (28), найдем фурье-амплитуду PKР $A_{\alpha \beta}(\tau)$ на неполносимметричном (недиагональном) колебании

$$
\begin{gathered}
A_{\alpha \beta}(\tau)=c_{k}^{(\alpha \beta)}\left(\bar{n}_{k}+1\right)^{1 / 2} e^{g_{0}(\tau)-g_{1}(\tau)} e^{i \tau \varepsilon_{\beta}} \times \\
X \int_{0}^{\tau} d s e^{i(\tau-s) \omega_{k}} e^{i s\left(\varepsilon_{\alpha}-\varepsilon_{\beta}\right)} e^{g_{1}(s)+g_{1}(\tau-s)} .
\end{gathered}
$$

Фурье-амплитуда РКР (32) определяется той же комбинацией g-функций, что и модулированный внешним возмущением спектр поглощения

$$
\begin{gathered}
x_{\alpha \beta}^{(1)}\left(\omega_{0}\right)=\frac{1}{2 \pi} \int_{-\infty}^{\infty} d \tau e^{-i\left(\omega_{0}-\varepsilon_{\beta}\right) \tau-\gamma_{\beta} \tau} F^{(1)}(\tau), \\
F^{(1)}(\tau)=e^{g_{0}(\tau)-g_{1}(\tau)} \int_{0}^{\tau} d s e^{g_{1}(s)+g_{1}(\tau-s)} e^{i s\left(\varepsilon_{\alpha}-\varepsilon_{\beta}\right)}
\end{gathered}
$$

(см. (17) и (29)-(31)). Отсюда следует, что функция

$$
\varphi(\tau)=e^{-g_{1}(\tau)} \int_{0}^{\tau} d s e^{g_{1}(s)+g_{1}(\tau-s)}=\frac{F^{(1)}(\tau)}{F^{(0)}(\tau)}
$$

может быть найдена делением фурье-образа спектра $x_{\alpha \alpha}^{(0)}$ на фурьеобраз спектра $x_{\alpha \beta}^{(1)}\left(F^{(0)}(\tau) / F^{(1)}(\tau)\right)$. Функцию $\exp \left(g_{1}(\tau)\right)$ можно вычислить, решая уравнение

$$
e^{g_{1}(\tau)}=\varphi^{-1}(\tau) \int_{0}^{\tau} d s e^{g_{1}(s)+g_{1}(\tau-s)} .
$$

Учитывая, что при малых $\tau$ эта функция известна

$$
\left(\exp \left(g_{1}(\tau)\right) \simeq\right.
$$

$\left.\simeq 1-i m_{1} \tau-\frac{m_{2}}{2} \tau^{2}\right)$, это интегральное уравнение можно решить численно методом последовательных приращений $\tau$. Одновременно с решением этого уравнения может быть найдена и фурье-амплитуда $A_{\alpha \beta}(\tau)$. Таким образом, путем только численной процедуры преобразования спектров $x_{\alpha \alpha}^{(0)}\left(\omega_{0}\right)$ и $x_{\alpha \beta}^{(1)}\left(\omega_{0}\right)$ можно определить $A_{\alpha \beta}(\tau)$ и, тем самым, возбуждающие профили РКР на недигональных неполносимметричных колебаниях.

Следует, однако, отметить, что процедура преобразования спектров поглощения в возбуждающие профили РКР для неполносимметричных недиагональных колебаний существенно сложнее, чем аналогичная процедура для полносимметричных и неполносимметричных диагональных колебаний. Кроме того, она требует использования не только обычного $x^{(0)}\left(\omega_{0}\right)$, но и модулированного внешним воздействием $x^{(1)}\left(\omega_{0}\right)$ спектра поглощения.

Полученную выше формулу (32) можно также использовать для модельных расчетов фурье-амплитуды РКР при заданных функциях $g_{0}(\tau)$ и $g_{1}(\tau)$. Для этого ее целесообразно преобразовать к фурьеинтегралу:

$$
A_{\alpha \beta}(\tau)=c_{k}^{(\alpha \beta)}\left(\bar{n}_{k}+1\right)^{1 / 2} e^{g_{0}(\tau)-g_{1}(\tau)} \int_{-\infty}^{\infty} d s e^{i s \omega_{k}} G(s) G(\tau-s),
$$




$$
G(x)=\theta(x) e^{g_{1}(x)},
$$

$\Theta(x)$ - ступенчатая функция Хэвисайда: $\Theta(x)=0$ при $x<0$, и $\Theta(x)=1$ при $x>0$. Интеграл (36) легко вычислить с помощью стандартных программ фурье-преобразования. Для получения возбуждающего профиля РКР следует еще раз провести фурье-преобразование функции $A_{\alpha \beta}(\tau)$ и возвести полученную амплитуду РКР в квадрат (см. (19)).

В частном случае слабого вибронного взаимодействия с диагональными неполносимметричными колебаниями (как, напр., $E_{g}$-колебаниями при кубической симметрии), когда можно приближенно взять $g_{1}(x) \simeq$ $\simeq 0$

$$
A_{\alpha \beta}(\tau)=c_{k}^{(\alpha \beta)}\left(\bar{n}_{k}+1\right)^{1 / 2} e^{g_{0}(\tau)}\left(e^{i \tau \omega_{k}}-1\right) e^{i \tau \varepsilon_{\alpha}} .
$$

(В предположении полного вырождения возбужденного электронного уровня $\varepsilon_{\alpha} \approx \varepsilon_{\beta}$.) Сравнивая эту формулу с формулами $(22)-(23)$, видим, что в случае слабого вибронного взаимодействия с неполносимметричными колебаниями амплитуда РКР на неполносимметричных колебаниях связана со спектром поглощения таким же соотношением, как и соответствующая амплитуда на полносимметричной моде.

Таким образом, в случае слабого взаимодействия как с недиагональными, так и с диагональными неполносимметричными колебаниями и при произвольном взаимодействии с полносимметричными колебаниями для PKР на всех модах применим обычный метод преобразования, основанный на формулах (22)-(23). Если взаимодействие с диагональными неполносимметричными колебаниями не мало́, то указанный метод справедлив только для полносимметричных и диагональных неполносимметричных колебаний. В этом случае для недиагональных неполносимметричных колебаний также существует процедура преобразования спектра поглощения в возбуждающий профиль РКР, однако она имеет более сложный характер (см. формулы $(32)-(36))$.

\section{ЛИТЕРАТУРА}

1. Jahn, H. A., Teller, E. // Proc. Roy. Soc., 1937, A161, 220.

2. Hizhnyakov, V., Tehver, I. // Proc. of the Second Intern. Conf. on Light Scattering in Solids. Paris, Flammarion, 1971, 57-61.

3. Hizhnyakov, V. V., Tehver, I. J. // Cooperative Phenomena. Proc. Intern. Symp. «Synergetics and Cooperative Phenomena in Solids and Macromolecules». Tallinn, Valgus, 1982, 133-144.

4. Hizhnyakov, V., Tehver, I. // Phys. Status solidi, 1967, 21, 755-768.

5. Tonks, D. L., Page, J. B. // Chem. Phys. Lett., 1979, 66, 449.

6. Champion, P. M., Albrecht, A. C. // Ann. Rev. Phys. Chem., 1982, 33, 353-376.

7. Ребане K. K., Хижняков В. В. Дополнение 2 в кн.: Бирман Дж. Пространственная симметрия и оптические свойства твердых тел. М., Мир, 1978, 327.

8. Feynman, R. P. // Phys. Rev., 1951, 84, 108.

9. Page, J. B., Tonks, D. L. // J. Chem. Phys., 1981, 75, 5694-5708.

10. Хижняков В. В. // Тр. ИФ АН ЭССР, 1986, № 59, 55-74.

11. Хиюняков В., Техвер И. // Изв. АН ЭССР. Физ. Матем., 1987, 36, № 3, 281288.

12. Hizhnyakov, V. V., Tehver, I. J. // Opt. Commun., 1980, 32, 419-421.

13. Tonks, D. L., Page, J. B. // Chem. Phys. Lett., 1981, 79, 247-252.

14. Tehver, I. J. // Opt. Commun., 1981, 38, 279-283.

15. Chan, C. K. // J. Chem. Phys., 1984, 81, 1614-1619.

16. Lu, H. M., Page, J. B. // Chem. Phys. Lett., 1986, 131, 87-93.

17. Lax, M. J. // Chem. Phys., 1952, 20, 1752 (см. перевод в кн. Проблемы физики полупроводников. М., ИЛ, 1957, 407-423). 


\section{HIŽNJAKOV, Imbi TEHVER}

\section{RESONANTS-KOMBINATSIOONHAJUMINE JAHN-TELLERI EFEKTI ARVESTADES: SEOS NEELDUMISEGA}

On arvutatud resonants-kombinatsioonhajumise Fourier' amplituudid kõrge sümmeetriaga lisanditsentrite (molekulide) tarvis kristallides (lahustes), mille ergastatud elektronseisundid on kõdunud. Niisugustes süsteemides tekib I järku hajumine ka mittetäissümmeetrilistel võnkumistel. Käesolevas töös piirdutakse nōrga vibrooninteraktsiooni juhuga mittetäissümmeetriliste vōnkumiste jaoks. Hajumise ergastusprofiilide modelleerimiseks on pakutud meetod, mille järgi need profiilid arvutatakse välisväljaga häiritud neeldumisspektri teisendamise teel. Saadud valemid on teisendusmeetodi üldistuseks kõdunud elektronseisundite puhul.

\section{HIZHNYAKOV and Imbi TEHVER}

\section{RESONANCE RAMAN SCATTERING, INCLUDING THE JAHN-TELLER EFFECT: CONNECTION WITH ABSORPTION}

The Fourier amplitudes of the resonance Raman scattering for impurity centres (molecules) in crystals (solutions), characterized by degenerate excited electronic states, are calculated. In such systems, the first-order Raman scattering by non-totally symmetric modes can be observed. Here the case of a weak vibronic interaction with non-totally symmetric modes is examined. A method is proposed to simulate Raman profiles by transforming the absorption spectrum perturbed by an external field. This is a generalization of the transform method for the case of degenerate electronic states. 